\title{
Monitoring of virgin coconut oil (VCO) adulteration with palm oil using fourier transform infrared spectroscopy.
}

\begin{abstract}
Virgin coconut oil (VCO) may be adulterated with cheaper oils, such as palm oil (PO). Thus, the detection and quantification of VCO adulteration with PO was monitored using Fourier transform infrared (FTIR) spectroscopy, combined with chemometrics of partial least square (PLS) and discriminant analysis at frequency regions of 3,010-3,000, 1,660-1,650 and $1,120-1,105 / \mathrm{cm}$. Attenuated total reflectance (ATR) measurements were made on pure VCO and that adulterated with varying concentrations of PO $(0.5-50 \% \mathrm{w} / \mathrm{w}$ in VCO). PLS calibration exhibited a good relationship between actual and FTIR-predicted values with coefficient of determination (R2) of 0.999 and standard error of calibration of 0.533 . The cross validation was performed by removing one standard at a time, and the final R2 value of 0.996 and standard error of prediction of 0.953 were obtained. The discriminant analysis using seven principal components was able to classify pure VCO and that adulterated with PO.
\end{abstract}

Keyword: Virgin coconut oil. 